DOI: http://dx.doi.org/10.5007/2175-7941.2014v31n1p156

\title{
A massa de ar e a sua determinação analítica ${ }^{+*}$
}

Guilherme de Almeida

Colégio Militar

Associação Portuguesa de Astrônomos Amadores (APAA)

Lisboa - Portugal

\section{Resumo}

A luz que nos chega de um astro tem de atravessar a atmosfera terrestre antes de chegar até nós. O comprimento do percurso efetuado por essa luz através da atmosfera é mínimo, quando o astro se encontra no zênite, coincidindo então com a espessura da própria atmosfera, mas aumenta à medida que o astro se aproxima do horizonte. Neste artigo mostraremos como calcular, por meio de um raciocínio geométrico, o quociente do comprimento do percurso da luz através da atmosfera pela espessura desta, valor conhecido como "massa de ar", em função da altura do astro.

Palavras-chave: Luz. Atmosfera. Refração. Índice de refração. Massa de ar. Horizonte. Altura de um astro. Zênite. Distância zenital.

\begin{abstract}
The light we receive from a celestial body has to cross the Earth atmosphere before to reach our eyes and telescopes. The path length of that light through the atmosphere is minimal when the celestial body is at zenith, equaling the atmosphere thickness itself, but it increases as the celestial body approaches horizon. In this
\end{abstract}

Air mass and its analytical derivation

* Recebido: junho de 2013.

Aceito: outubro de 2013. 
article we will show how to derivate the quotient of the light path length and the atmosphere thickness (quantity usually known as "air mass"), as a function of the celestial body altitude.

Keywords: Light. Atmosphere. Refraction. Refraction index. Air mass. Horizon. Altitude of a celestial body. Zenith. Zenital distance.

\section{Introdução}

É sabido que a luz que nos chega de um astro tem de atravessar a atmosfera terrestre antes de chegar até nós. O comprimento do percurso $(d)$ efetuado por essa luz através da atmosfera depende principalmente da altura $h$ a que esse astro se encontra no momento da observação. Tal comprimento é mínimo, e coincide com a espessura da própria atmosfera $(H)$, quando o astro se encontra no zênite e aumenta à medida que o astro se aproxima do horizonte. Neste artigo, propomonos calcular, por meio de um raciocínio geométrico, o comprimento do percurso $d$, relativamente a $H$, para diversos valores de $h$.

\section{Conceitos fundamentais}

O zênite é o ponto imaginário do céu, na vertical, por cima da cabeça do observador. Em Astronomia, dá-se habitualmente o nome de massa de ar, T, (ou coeficiente de massa de ar) ao quociente $T=d / H$. Assim sendo, $T=1$ para um astro no zênite (ou seja, $h=90^{\circ}$ ) e é cada vez maior à medida que a altura $h$ do astro diminui. Muitas vezes, considera-se a distância zenital (z) do astro, ou seja, a distância angular entre esse astro e o zênite (Fig. 1). Como $z+h=90^{\circ}$, então, $z=90^{\circ}-h$.

\section{Aproximação da atmosfera plano-paralela}

Para calcular a massa de ar, utiliza-se frequentemente uma aproximação, que consiste em considerar, na vizinhança imediata da região onde o observador se encontra, a Terra como se fosse plana, assim como a sua atmosfera: é a "aproximação plano-paralela", a que poderíamos chamar "aproximação da Terra plana". Da Fig. 1 concluímos facilmente que 


$$
\cos z=\frac{H}{d}
$$

e sabemos que

$$
T=\frac{d}{H}
$$

Consequentemente, $T=\frac{1}{\cos z}$

(Equação 1),

onde $z$ é a distância zenital do astro observado. Esta aproximação é válida para valores de $z$ até cerca de $75^{\circ}$, ou seja, para astros a alturas acima de $15^{\circ}$. Para alturas menores (distâncias zenitais maiores), a fórmula deixa de dar resultados aceitáveis, devido à curvatura da Terra e à atmosfera.

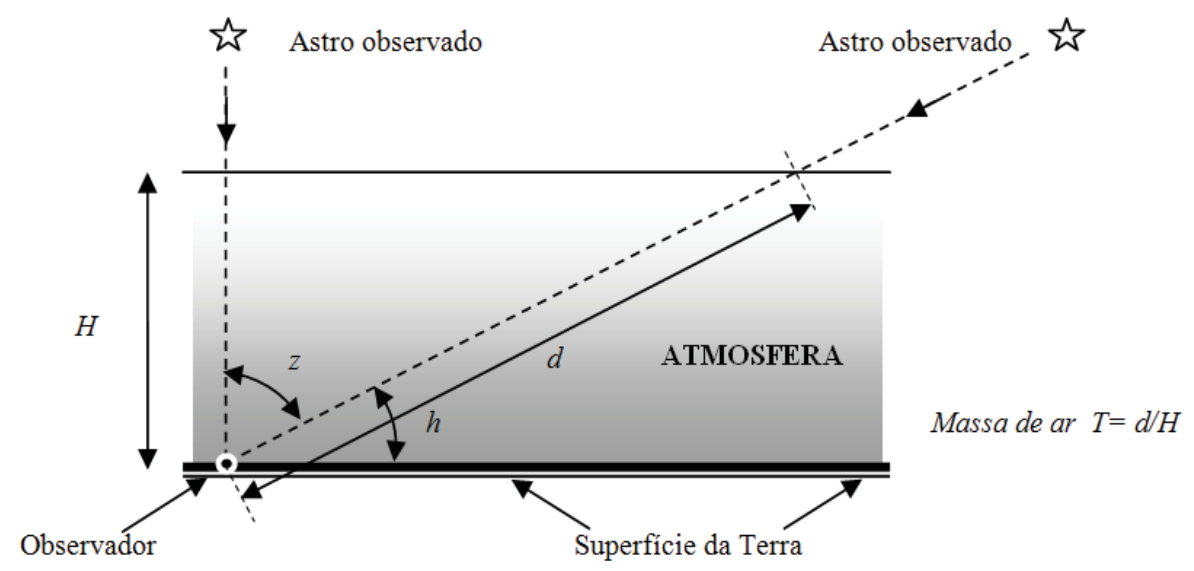

Fig. 1 - Atmosfera e massa de ar na aproximação da atmosfera "planoparalela" ou da "Terra plana". A refração atmosférica não foi representada, por ser muito pequena à escala da figura $\left(h>15^{\circ}\right)$. Para maior clareza, a distância do astro e a espessura da atmosfera não foram obviamente representadas em escala (Figura do autor). 
A refração atmosférica produz um ligeiro aumento da altura aparente dos astros (veja-se a nota final 1). É nula no zênite, crescendo com a distância zenital $z$ : vale cerca de $0,26^{\prime}$ para $z=20^{\circ}\left(h=70^{\circ}\right), 1^{\prime}$ para $z=45^{\circ}\left(h=45^{\circ}\right), 2,8^{\prime}$ para $z=70^{\circ}$ $\left(h=20^{\circ}\right)$ e cerca de meio grau (34') para o horizonte, onde $z=90^{\circ}\left(h=0^{\circ}\right)$. Assim sendo, a altura aparente de um astro é geralmente pouco superior à sua altura verdadeira, mas dado que essa diferença é, em geral, muito pequena, como acabamos de referir, desprezaremos a refração no cálculo de $d$.

Além disso, a refração atmosférica produz um percurso ligeiramente curvo da luz através da atmosfera terrestre, dado que o ar não é um meio homogêneo e o seu índice de refração cresce no sentido das baixas altitudes (veja-se a nota final 2). No entanto, o seu efeito no alongamento dos percursos $d$ é reduzido: basta pensar que, ao nível do mar (onde é máximo), o índice de refração do ar, $n_{\mathrm{ar}}=1,00029$, é mais elevado a baixas altitudes e que, para o vácuo, temos $n_{0}=1,00000$. Como a curvatura dos raios luminosos através da atmosfera é muito pequena, o seu trajeto pode ser, sem grande erro, aproximado por um segmento de reta.

\section{Um cálculo mais geral, para qualquer valor de $\boldsymbol{h}$}

Para alturas inferiores a $15^{\circ}$, existem diversas equações especiais para o cálculo de $d$, de diversos investigadores (YOUNG \& IRVINE, HARDIE, PICKERING, KASTEN \& YOUNG, ROZENBERG), em geral muito complexas. Apresento seguidamente a equação que deduzimos independentemente, bem mais simples, mas que dá resultados muito próximos dos obtidos pelas equações mais complexas.

Aplicando o teorema de Carnot ao triângulo [COP] da Fig. 2, obtemos

$$
(R+H)^{2}=R^{2}+d^{2}-2 R d \cos \left(180^{\circ}-z\right),
$$

que também podemos escrever como

$$
(R+H)^{2}=R^{2}+d^{2}+2 R d \cos z,
$$

$R$ é o raio terrestre médio e foi considerado $R=6371 \mathrm{~km}$.

Desenvolvendo e simplificando (e, como $z+h=90^{\circ}$ ), obtemos

$$
H^{2}+2 R H=d^{2}+2 R d \sin h,
$$

ou seja,

$$
d^{2}+(2 R \sin h) d-\left(H^{2}+2 R H\right)=0
$$

que os leitores atentos reconhecerão como a clássica equação do $2 .^{\circ}$ grau, em ordem a $d$, com os coeficientes $a=1, b=2 R \sin h e c=-\left(H^{2}+2 R H\right)$. 


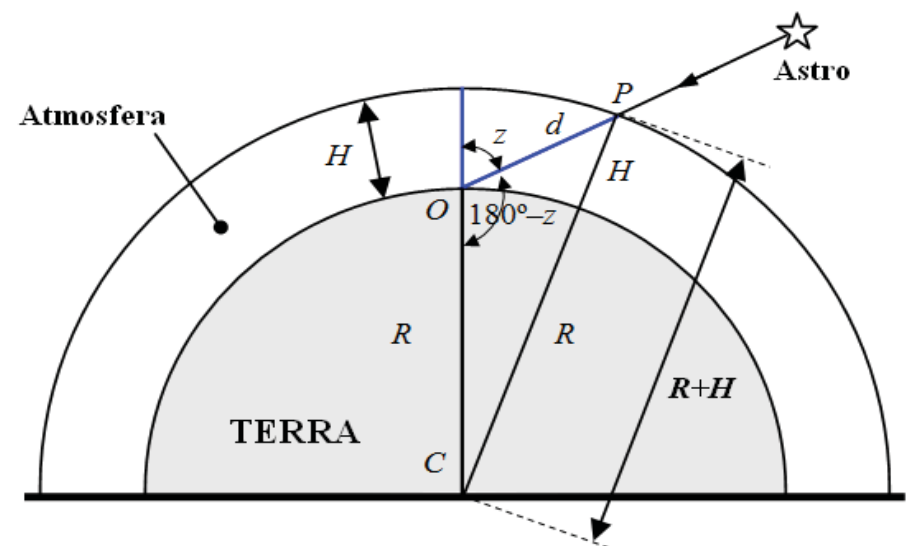

Fig. 2 - Figura esquemática para a determinação de d e de T, considerando a curvatura da Terra e da atmosfera. O significado dos símbolos incluídos na figura está referido no texto. Para maior clareza, a distância do astro e a espessura da atmosfera não foram obviamente representadas à escala (Figura do autor).

A fórmula resolvente conduz a duas soluções, mas a solução positiva, a única que nos interessa, vale:

$$
d=\frac{-2 R \sin h+\sqrt{4 R^{2} \sin ^{2} h+4 \times 1\left(H^{2}+2 R H\right)}}{2},
$$

que, após simplificação, nos leva a

$$
d=-R \sin h+\sqrt{R^{2} \sin ^{2} h+H^{2}+2 R H} .
$$

Esta equação fornece a distância $d$ para qualquer $h$. A massa de ar, definida como $T=d / H$ valerá pois

$$
T=\frac{d}{H}=\frac{-R \sin h+\sqrt{R^{2} \sin ^{2} h+H^{2}+2 R H}}{H}
$$

Podemos ver facilmente que, para um astro no zênite $\left(h=90^{\circ}\right)$, esta equação dá $T=1$, como seria de esperar (neste caso, $d=H$ ). Para um astro no horizonte $\left(h=0^{\circ}\right)$, obtemos

$$
T=\frac{d}{H}=\frac{0+\sqrt{0+H^{2}+2 R H}}{H}
$$


ou seja, após simplificação,

$$
T=\sqrt{1+\frac{2 R}{H}} .
$$

A equação 3 mostra claramente que o valor de $T$ depende da espessura $H$ da atmosfera, sempre que não se possa aplicar a aproximação da atmosfera planoparalela referida na Fig 1. E o valor de $H$ é especialmente relevante para astros a alturas muito baixas, incluindo o caso do astro no horizonte geométrico, como se evidencia na equação 4 .

Torna-se, pois, necessário calcular a espessura da atmosfera, $H$, para podermos prosseguir neste cálculo e obter valores concretos da massa de ar para astros a qualquer altura. No entanto, esse cálculo não é imediato, porque o limite superior da atmosfera terrestre não é vincado e esta esbate-se progressivamente, sem existir um limite superior nítido. Devido a este fato, a medida da espessura $H$ da atmosfera depende do critério usado para definir esse limite. Como estabelecer tal limite? Por exemplo, no topo do monte Everest (8848 m), a pressão atmosférica vale cerca de $1 / 3$ da que se verifica ao nível do mar; no entanto, a $300 \mathrm{~km}$ de altitude ainda se detectam vestígios da atmosfera, embora esta seja já muito tênue.

\section{IV.1 Critério para a determinação do valor $\boldsymbol{H}$}

Para calcular a espessura da atmosfera, comecemos por recordar que a pressão atmosférica e a densidade do ar diminuem com a altitude: por exemplo, a $8500 \mathrm{~m}$ de altitude a densidade do ar é apenas $35 \%$ da que se verifica ao nível do mar; a 11,6 km de altitude cai para cerca de 1/8 do valor ao nível do mar. A temperatura também varia com a altitude. Ao nível do mar e a $15^{\circ} \mathrm{C}(288,15 \mathrm{~K})$, a pressão atmosférica padrão vale $101325 \mathrm{~Pa}$ e a densidade do ar é $1,2250 \mathrm{~kg} / \mathrm{m}^{3}$. Um método de cálculo bastante útil consiste numa aproximação em que se raciocina como se a atmosfera terrestre fosse homogênea e isotérmica, com os valores da pressão e da temperatura iguais aos que se verificam, em média, ao nível do mar: $\rho_{\mathrm{ar}}=1,2250 \mathrm{~kg} / \mathrm{m}^{3} ; T=288,15 \mathrm{~K} ; p_{0}=101325 \mathrm{~Pa}$, com a aceleração da gravidade padrão, $g=9,80665 \mathrm{~m} / \mathrm{s}^{2}$. Por considerações hidrostáticas, vamos determinar que altura deveria ter a atmosfera, suposta homogênea a isotérmica, para produzir a pressão observada ao nível do mar:

$p=\rho g H \Leftrightarrow 101325=1,2250 \times 9,80665 \times H \Leftrightarrow H=\frac{101325}{1,2250 \times 9,80665}=8434,5 \mathrm{~m}$. 
Concluímos que, se toda a atmosfera estivesse nas condições de temperatura e pressão referidas, com $g$ constante, todo o ar estaria contido numa espessura de cerca de $8,4 \mathrm{~km}$. Sabemos que a atmosfera mede mais de $8,4 \mathrm{~km}$ de altitude e que $g$ diminui com a altitude. Assim, o valor a que chegamos (os $8,4 \mathrm{~km}$ ) parece, à primeira vista, um absurdo e uma expectativa demasiado baixa. Mas outros fatos jogam a nosso favor:

a) embora a atmosfera vá além dos $8,4 \mathrm{~km}$, nas altitudes superiores, ela é pouco densa, pelo que as partes mais altas da atmosfera contribuem pouco para a sua massa.

b) estamos a admitir que desde o nível do mar até aos $8,4 \mathrm{~km}$ de altitude, a densidade do ar se mantém, mas na realidade ela baixa;

c) ao considerarmos a situação b), estivemos a admitir que, por exemplo, a $6 \mathrm{~km}$ de altitude a densidade do ar é igual à que se verifica ao nível do mar, pelo que a não consideração do ar a mais de $8,4 \mathrm{~km}$ de altitude é contrariada pela incorporação no cálculo de uma densidade maior do que a real nas altitudes até $8,4 \mathrm{~km}$;

d) a variação de $g$ é muitíssimo pequena para altitudes desta grandeza $\left(\Delta g / g_{0} \approx 0,26 \%\right)$.

Dados estes pressupostos, e para a finalidade específica da determinação da massa de ar, a suposição simplificadora acima referida é por isso razoável e seguida por muitos autores, tal como aqui faremos (veja-se a nota final 3). Se na equação 3 , considerarmos $H=8,4 \mathrm{~km}$ e, por exemplo, para um astro à altura $h=20^{\circ}$, obteremos $T=2,91$. Com a equação 4 , resulta $T=39,0$ para um astro no horizonte. A equação 3 é mais geral que a equação 1. Embora a equação 3 ainda seja uma simplificação, não considerando os efeitos da refração, aliás, muito pequenos para alturas acima dos $45^{\circ}$ (como já referimos), os seus resultados são muito satisfatórios. Tal acontece porque o aumento do comprimento de percurso $(d)$ da luz na atmosfera, causado pelo encurvamento da luz devido à refração, é um aumento muito pequeno. Ou seja, o percurso curvo (atendendo à refração) é pouco maior (com diferença minúscula) do que o percurso retilíneo na aproximação que aqui consideramos.

\section{IV.2 Resultados obtidos}

Os resultados obtidos para a massa de ar relativamente a astros a diversas alturas desde $90^{\circ}$ (zênite) até $0^{\circ}$ (no horizonte geométrico), utilizando a equação 3 , são indicados na tabela 1 . 
Tabela 1 - Massa de ar para diferentes alturas (segundo a equação 3) (considerando $H=8,4 \mathrm{~km}$ e o raio terrestre médio $R=6371 \mathrm{~km}$ ).

\begin{tabular}{|c|c|c|c|c|c|}
\hline Altura $h$ & $\begin{array}{c}\text { Massa de ar } \\
T\end{array}$ & Altura $h$ & $\begin{array}{c}\text { Massa de ar } \\
T\end{array}$ & Altura $h$ & Massa de ar \\
\hline $90^{\circ}$ & 1,00 & $55^{\circ}$ & 1,22 & $20^{\circ}$ & 2,91 \\
\hline $85^{\circ}$ & 1,00 & $50^{\circ}$ & 1,30 & $15^{\circ}$ & 3,83 \\
\hline $80^{\circ}$ & 1,02 & $45^{\circ}$ & 1,41 & $10^{\circ}$ & 5,64 \\
\hline $75^{\circ}$ & 1,04 & $40^{\circ}$ & 1,55 & $5^{\circ}$ & 10,63 \\
\hline $70^{\circ}$ & 1,06 & $35^{\circ}$ & 1,74 & $3^{\circ}$ & 15,92 \\
\hline $65^{\circ}$ & 1,10 & $30^{\circ}$ & 2,00 & $1^{\circ}$ & 27,91 \\
\hline $60^{\circ}$ & 1,15 & $25^{\circ}$ & 2,36 & $0^{\circ}$ & 38,96 \\
\hline
\end{tabular}

A tabela 1 permita avaliar melhor as limitações do modelo de atmosfera plano-paralela (equação 1). Para $h=60^{\circ}\left(z=30^{\circ}\right)$, a aproximação plano-paralela da Fig. 1, daria $T=1,15$, o que é satisfatório e em boa concordância com a Tabela 1; mas para $h=15^{\circ}\left(z=75^{\circ}\right)$, já se obteria $T=3,86$, valor menos satisfatório; e, pior ainda, para $h=5^{\circ}\left(\mathrm{z}=85^{\circ}\right)$ tal aproximação daria $T=11,47$. Para $h=0^{\circ}$, a equação 1 faria a massa de ar tender para infinito!

A tabela 1 e o gráfico da Fig. 3 mostram a importância capital de se observar com a menor distância zenital possível (máxima altura). Por exemplo, ao observar um planeta a $30^{\circ}$ de altura, ele é visto através de uma espessura de ar que será o dobro da que seria se o planeta estivesse no zênite. Ou mais de dez vezes superior (comparada com a do zênite) se o astro se encontrar a $5^{\circ}$ de altura.

Mas, em geral, os objetos que observamos não estão perto do zênite. Mesmo assim, veja-se que observando um astro junto ao horizonte $\left(h=5^{\circ}\right)$, a espessura de ar é mais de sete vezes a que será para $h=45^{\circ}$. Isso faz-se notar tanto na turbulência como na extinção. E também na refração diferencial que veremos num próximo artigo.

Os resultados obtidos com a equação 3 são bastante satisfatórios e muito aproximados aos das fórmulas mais complexas, obtidas por diferentes investigadores. A comparação entre os valores propostos para $T$, para o caso mais difícil e complicado $\left(h=0^{\circ}\right)$, encontra-se na tabela 2 . 


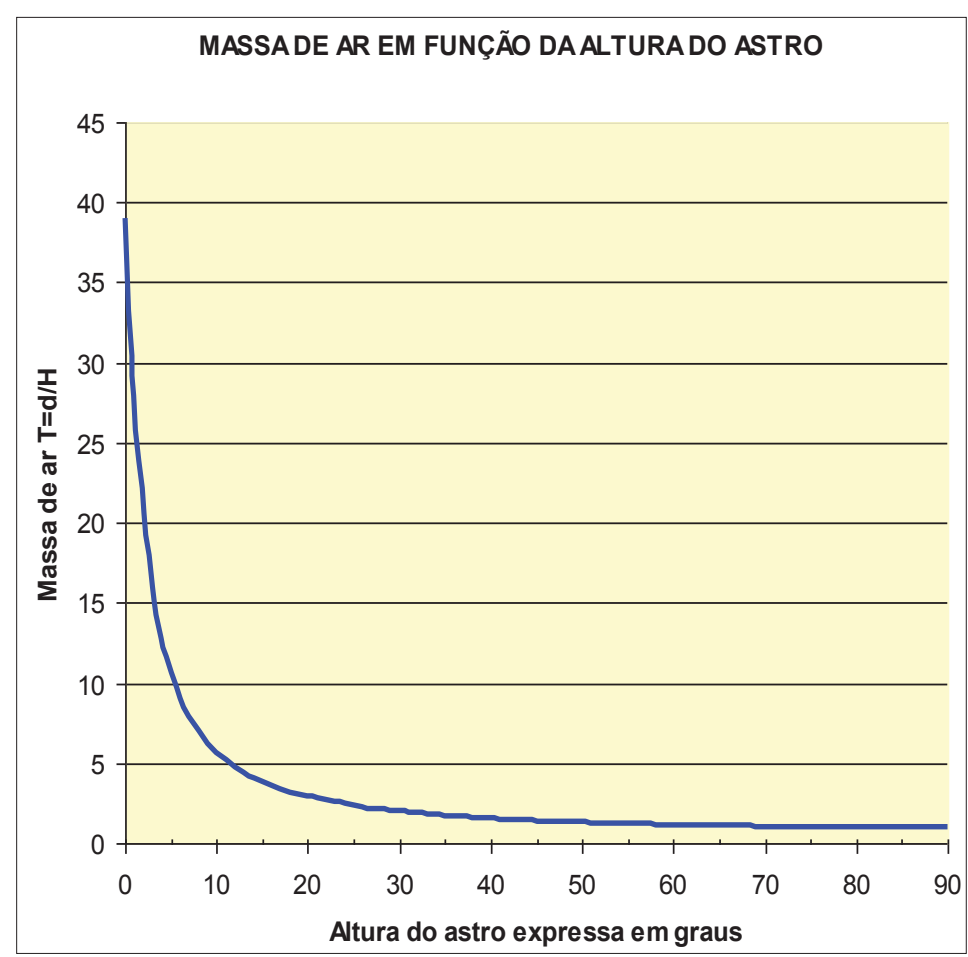

Fig. 3 - Representação gráfica da dependência de $T$ com $h$, utilizando a equação 3 (Figura do autor).

Tabela 2 - Comparação entre os valores da massa de ar para um astro no horizonte, segundo diferentes autores.

\begin{tabular}{|l|c|c|c|c|c|}
\hline $\begin{array}{l}\text { Autor } \\
\text { (data) }\end{array}$ & $\begin{array}{c}\text { Guilherme } \\
\text { de Almeida } \\
(2010)\end{array}$ & $\begin{array}{c}\text { Rozenberg } \\
(1966)\end{array}$ & $\begin{array}{c}\text { Kasten \& } \\
\text { Young } \\
(1989)\end{array}$ & $\begin{array}{c}\text { M. Kocifaj } \\
\text { (1995) }\end{array}$ & $\begin{array}{c}\text { Pickering } \\
\text { (2002) }\end{array}$ \\
\hline $\begin{array}{l}\text { Determi- } \\
\text { nação de } T \\
\text { para } h=0^{\circ}\end{array}$ & $T=39,0$ & $T=40$ & $T=38$ & $T=40,4$ & $T=38,7$ \\
\hline
\end{tabular}

Em regra, para obter imagens pouco degradadas pela atmosfera, evitam-se observações astronômicas de astros a baixa altura $\left(h<20^{\circ}\right)$. Porém, há casos em 
que não há outro remédio, quando da conjugação da declinação do astro com a latitude do lugar de observação, resultar que a altura máxima atingida por este seja escassa (veja a nota final 4) e o astro não se eleve tanto como o observador desejaria. O caso $h=0^{\circ}$ é uma solução extrema de interesse apenas ligado ao cálculo geométrico, mas tal não significa que um astro no horizonte se preste a boas observações.

(1) O raio luminoso vindo do zênite atravessa as camadas atmosféricas perpendicularmente, não sofrendo por isso desvio da sua direção inicial. Um raio luminoso oblíquo, ao passar por regiões da atmosfera de índice de refração cada vez maior, refrata-se, aproximando-se da normal, pelo que (para a observador) a luz do astro parece vir de uma posição a uma altura ligeiramente maior do que se não houvesse atmosfera. Chama-se altura verdadeira à que se obteria se não houvesse atmosfera e altura aparente à que o observador verifica. $\mathrm{O}$ valor desse desvio também depende do comprimento de onda da luz, sendo maior para a luz de menores comprimentos de onda.

(2) Dado que o índice de refração do ar é gradualmente crescente à medida que a luz se aproxima da Terra, o trajeto dos raios luminosos é também ligeiramente encurvado. Neste artigo, pretende-se calcular o comprimento do percurso $d$ por métodos geométricos, sem olhar à refração atmosférica. Em um próximo artigo, estes efeitos e outros igualmente interessantes serão considerados em detalhe.

(3) Cerca de $65 \%$ da massa da atmosfera encontra-se abaixo desta altitude. Na nossa aproximação, tudo se passa como se tivéssemos concentrado toda a massa da atmosfera $\left(5,148 \times 10^{18} \mathrm{~kg}\right)$ numa espessura de $8,4 \mathrm{~km}$. Tal não significa que o valor $H=8,4 \mathrm{~km}$ seja admissível para outras considerações (por exemplo, para a aeronáutica). E também não significa que a espessura da atmosfera real seja apenas de $8,4 \mathrm{~km}$.

(4) Para obter informação sobre estes conceitos, e sobre a altura máxima do astro em função da sua declinação e da latitude do observador, pode consultar-se a obra FERREIRA, M.; ALMEIDA, G. Introdução à Astronomia e às Observações Astronómicas. 7. ed. Lisboa: Plátano Editora, 2004. 


\section{Referências:}

ALMEIDA, G.; RÉ, P. Observar o Céu Profundo. 2. ed. Lisboa: Plátano Editora, 2003.

Mais informação sobre os autores referidos no início da seção 3 (e na tabela 2), acessível em:

$<$ http://en.wikipedia.org/wiki/Airmass $>$

$<$ http://www.starlink.rl.ac.uk/docs/sc6.htx/node15.html>

$<$ http://en.wikipedia.org/wiki/Air_mass_\%28astronomy\%29>.

KOCIFAJ, M. Optical air mass and refraction in a Rayleigh atmosphere. Astronomical Institute of Slovak Academy of Sciences, 1995. Disponível em: $<$ http://www.ta3.sk/caosp/Eedition/FullTexts/vol26no1/pp23-30.pdf>. 